\title{
Front Matter: Volume 9817
}

, "Front Matter: Volume 9817," Proc. SPIE 9817, Seventh International Conference on Graphic and Image Processing (ICGIP 2015), 981701 (15 December 2015); doi: 10.1117/12.2230991

SPIE Event: Seventh International Conference on Graphic and Image Processing, SPIE. 2015, Singapore, Singapore 
PROCEEDINGS OF SPIE

\title{
Seventh International Conference on Graphic and Image Processing (ICGIP 2015)
}

\author{
Yulin Wang \\ Xudong Jiang \\ Editors
}

23-25 October 2015

Singapore, Singapore

Organized by

International Association of Computer Science and Information Technology (Singapore)

Sponsored by

Wuhan University (China)

Published by

SPIE 
The papers in this volume were part of the technical conference cited on the cover and title page. Papers were selected and subject to review by the editors and conference program committee. Some conference presentations may not be available for publication. Additional papers and presentation recordings may be available online in the SPIE Digital Library at SPIEDigitallibrary.org.

The papers reflect the work and thoughts of the authors and are published herein as submitted. The publisher is not responsible for the validity of the information or for any outcomes resulting from reliance thereon.

Please use the following format to cite material from these proceedings:

Author(s), "Title of Paper," in Seventh International Conference on Graphic and Image Processing (ICGIP 2015), edited by Yulin Wang, Xudong Jiang, Proceedings of SPIE Vol. 9817 (SPIE, Bellingham, WA, 2015) Six-digit Article CID Number.

ISSN: 0277-786X

ISSN:1996-756X (electronic)

ISBN: 9781510600584

Published by

SPIE

P.O. Box 10, Bellingham, Washington 98227-0010 USA

Telephone +1 3606763290 (Pacific Time) · Fax +1 3606471445

SPIE.org

Copyright (C) 2015, Society of Photo-Optical Instrumentation Engineers.

Copying of material in this book for internal or personal use, or for the internal or personal use of specific clients, beyond the fair use provisions granted by the U.S. Copyright Law is authorized by SPIE subject to payment of copying fees. The Transactional Reporting Service base fee for this volume is $\$ 18.00$ per article (or portion thereof), which should be paid directly to the Copyright Clearance Center (CCC), 222 Rosewood Drive, Danvers, MA 01923. Payment may also be made electronically through CCC Online at copyright.com. Other copying for republication, resale, advertising or promotion, or any form of systematic or multiple reproduction of any material in this book is prohibited except with permission in writing from the publisher. The CCC fee code is $0277-786 \mathrm{X} / 15 / \$ 18.00$.

Printed in the United States of America.

Publication of record for individual papers is online in the SPIE Digital Library.

\section{SPIE. DIGITAL}

Paper Numbering: Proceedings of SPIE follow an e-First publication model. A unique citation identifier (CID) number is assigned to each article at the time of publication. Utilization of CIDs allows articles to be fully citable as soon as they are published online, and connects the same identifier to all online and print versions of the publication. SPIE uses a six-digit CID article numbering system structured as follows:

- The first four digits correspond to the SPIE volume number.

- The last two digits indicate publication order within the volume using a Base 36 numbering system employing both numerals and letters. These two-number sets start with $00,01,02,03,04$, $05,06,07,08,09,0 A, O B \ldots$ OZ, followed by 10-1Z, 20-2Z, etc. The CID Number appears on each page of the manuscript. 


\title{
Contents
}

\author{
vii Authors \\ ix Conference Committee \\ xi Introduction
}

SESSION 1 IMAGE ANALYSIS AND IMAGE ENHANCEMENT

981702 Block based image compression technique using rank reduction and wavelet difference reduction [9817-6]

981703 A novel targeted enhancement technique for decimation and interpolation of images [9817-7]

981704 Spectral residual method of saliency detection based on the two-dimensional fractional Fourier transform domain [9817-12]

981705 An advanced total variation model in $\mathrm{H}^{-1}$ space for image inpainting [9817-57]

981706 Image enhancement based on edge boosting algorithm [9817-24]

981707 Learning the histogram sequences of generalized local ternary patterns for blind image quality assessment [9817-25]

$981708 \quad$ Error image aware content restoration [9817-43]

\section{SESSION 2 IMAGE PROCESSING AND APPLICATION}

981709 Game theory based band selection for hyperspectral images [9817-3]

$98170 \mathrm{~A}$ Hardware accelerator of convolution with exponential function for image processing applications [9817-10]

9817 OB 3D CAD model retrieval method based on hierarchical multi-features [9817-33]

$98170 \mathrm{C}$ A novel edge-preserving nonnegative matrix factorization method for spectral unmixing [9817-34]

9817 OD An improved adaptive IHS method for image fusion [9817-37]

9817 OE Spatial and spectral coordinate super resolution of hyperspectral imagery based on redundant dictionary [9817-30] 
9817 OF Brain tumor segmentation in MR slices using improved GrowCut algorithm [9817-23]

9817 OG Hippocampus segmentation using locally weighted prior based level set [9817-27]

$9817 \mathrm{OH}$ Automatic and efficient methods applied to the binarization of a subway map [9817-35]

9817 ol A graph-based watershed merging using fuzzy C-means and simulated annealing for image segmentation [9817-39]

$98170 \mathrm{~J}$ Automatic segmentation and classification of tendon nuclei from IHC stained images [9817-48]

9817 OK Automatic segmentation of Leishmania parasite in microscopic images using a modified CV level set method [9817-49]

9817 OL Adaptive segmentation of nuclei in H\&S stained tendon microscopy [9817-53]

$98170 \mathrm{M}$ A novel iris segmentation algorithm based on small eigenvalue analysis [9817-40]

\section{SESSION 4 IMAGE DETECTION AND PATTERN RECOGNITION}

9817 ON Distance-invariant automatic engagement level recognition using visual cues [9817-2]

981700 Learning deformation model for expression-robust 3D face recognition [9817-9]

9817 OP An improved space-based algorithm for recognizing vehicle models from the side view [9817-17]

$98170 Q$ Object recognition based on Google's reverse image search and image similarity [9817-41]

9817 OR A novel ensemble method to integrate with different technologies for moving foreground detection [9817-44]

9817 OS A real-time marking defect inspection method for IC chips [9817-54]

9817 OT Fast frequency domain method to detect skew in a document image [9817-31]

9817 OU A small dim infrared maritime target detection algorithm based on local peak detection and pipeline-filtering [9817-32]

9817 OV Detection and clustering of features in aerial images by neuron network-based algorithm [9817-56]

\section{SESSION 5 COMPUTER VISION AND VISUALIZATION}

9817 OW Robust visual track using an ensemble cascade of convolutional neural networks [9817-8]

iv 
$98170 X \quad$ Robust visual tracking with dual spatio-temporal context trackers [9817-11]

9817 OY Stereo vision-based pedestrian detection using multiple features for automotive application [9817-16]

$98170 Z$ Action recognition based on a selective sampling strategy for real-time video surveillance [9817-19]

981710 Multi-expert tracking algorithm based on improved compressive tracker [9817-22]

981711 Development of embedded real-time and high-speed vision plafform [9817-46]

\section{SESSION 6 COMPUTATIONAL PHOTOGRAPHY AND AIDED TECHNOLOGY}

981712 Performance evaluation and optimization of BM4D-AV denoising algorithm for cone-beam CT images [9817-15]

981713 Impact of memory bottleneck on the performance of graphics processing units [9817-51]

981714 A non-linear camera calibration with modified teaching-learning-based optimization algorithm [9817-52]

981715 Optimizations of the GPU-based three-dimensional FDTD program with CPML boundary condition on Kepler architecture GPU [9817-58]

\section{SESSION 7 FILTER DESIGN AND APPLICATION}

981716 Ship detection from high-resolution imagery based on land masking and cloud filtering [9817-20]

981717 A new mixed-mode filter based on MDDCCs [9817-38]

981718 Comparison of recent non-linear filters from graphics field [9817-47]

\section{SESSION 8 REMOTE SENSING AND IMAGE FORMATION}

981719 Ultrasound harmonic imaging with reducing speckle noise by spatial-frequency compounding approach [9817-1]

9817 1A Four decades urban growth and land use change in Samara Russia through remote sensing and GIS techniques [9817-5]

9817 1B An adjustable frame-straddling image formation system for PIV application [9817-45]

9817 1C Remote sensing image classification based on support vector machine with the multi-scale segmentation [9817-14] 
9817 1D Application of linear graph embedding as a dimensionality reduction technique and sparse representation classifier as a post classifier for the classification of epilepsy risk levels from EEG signals [9817-4]

9817 1E The serial message-passing schedule for LDPC decoding algorithms [9817-13]

$9817 \mathrm{IF}$ Iterative feedback algorithm for phase retrieval based on transport of intensity equation [9817-42] 


\title{
Authors
}

Numbers in the index correspond to the last two digits of the six-digit citation identifier (CID) article numbering system used in Proceedings of SPIE. The first four digits reflect the volume number. Base 36 numbering is employed for the last two digits and indicates the order of articles within the volume. Numbers start with 00, 01, 02, 03, 04, 05, 06, 07, 08, 09, OA, OB...0Z, followed by 10-1Z, 20-2Z, etc.

\author{
Abdullah, Rosni, 0l \\ Achuthan, Anusha, OG \\ An, Ran, $\mathrm{OB}$ \\ Anbarjafari, Gholamreza, 02 \\ Aruna Kumar, S. V., OM \\ Bao, Wenxing, OC, IC \\ Bolotnikova, Anastasia, 02 \\ Boori, Mukesh Singh, 1 A \\ Bucha, Victor, OA \\ Chan, Yi-Tung, OR \\ Chen, Liang, OF \\ Chen, Wen-Pin, OR \\ Cheng, Dansong, 05 \\ Cheng, Hong, $1 \mathrm{~F}$ \\ Chitsobhuk, Orachat, 06 \\ Choi, Hong Jun, 13 \\ Choi, Sungwoo, 08 \\ Choudhary, Komal, 1A \\ Chuang, Bo-I, OL \\ Daneshmand, Morteza, 02 \\ Ding, Youdong, OP \\ Dong, Lili, OU \\ Dong, Yimin, 11 \\ Du, Songlin, 07 \\ Durand, Philippe, $\mathrm{OH}$ \\ Dutta, Maitreyee, OT \\ Ensafi, Shahab, OK \\ Farahi, Maria, OK \\ Feng, Wei, 1C \\ Feng, Yachun, 10 \\ Fu, Kun, 18 \\ Ghorbanzadeh, Dariush, $\mathrm{OH}$ \\ Guo, Wei, 19 \\ Guo, Zhe, 00 \\ Guru, D. S., OM \\ Harish, B. S., OM \\ He, Zhenyu, 09 \\ Horváth, András, $O Q$ \\ Hou, Zhiqiang, OW \\ Hsu, Jian-Han, OL \\ Hu, Dan, OW \\ $\mathrm{Hu}$, Yang, OS \\ Huang, Fengchen, 09 \\ Huang, Kuidong, 12 \\ Jaupi, Luan, $\mathrm{OH}$ \\ Ji, Chunhong, OF \\ Jiang, Shuangshuang, 17 \\ Jiang, Xue, $1 \mathrm{E}$ \\ Jin, Tianming, 16
}

\author{
Jou, I-Ming, OJ, OL \\ Jung, Byunghee, 08 \\ Kim, Cheol Hong, 13 \\ Kim, Dongyoung, OY \\ Kim, Jaehong, ON \\ Kim, Jong Myon, 13 \\ Kovelskiy, Viktor, $1 \mathrm{~A}$ \\ Kuok, Chan-Pang, OJ \\ Kupriyanov, Alexander, 1A \\ Lee, Chung-Hee, OY \\ Lee, Dongjin, ON \\ Lee, Moonsik, 08 \\ Lei, Tao, 00 \\ $\mathrm{Li}$, Rong, OP \\ Liang, Xiao, 17 \\ Lin, Pei-Ru, OR \\ Linton, David, 15 \\ Liu, Kaifeng, $1 \mathrm{~F}$ \\ Liu, Lin, 05 \\ Liv, Mingshan, $1 \mathrm{E}$ \\ Liu, Shanshan, 1E \\ Liu, Shu, 00 \\ Lusi, liris, 02 \\ Ma, Ruishi, 0C, 1C \\ Ma, Yide, 07 \\ Mao, Ying, OF \\ Mehta, Sunita, OT \\ Ngernplubpla, Jaturon, 06 \\ $\mathrm{Ngo}$, Minh $\mathrm{Ngoc}, \mathrm{OM}$ \\ Noroozi, Fatemeh, 02 \\ Ouyang, Zhenxing, 11, 1B \\ Panchenko, Ivan, OA \\ Park, Chankyu, ON \\ Poonacha, P. G., 03 \\ Prabhakar, Sunil Kumar, ID \\ Prabhakaran, P. Joy, 03 \\ Qi, Lin, 04 \\ Rabbani, Hossein, OK \\ Rajaguru, Harikumar, 1D \\ Rajeswari, Mandava, 0G, Ol \\ Rasti, Pejman, 02 \\ Samuel, Kadri, 02 \\ Sarkar, Suman, 02 \\ Sarrafzadeh, Omid, OK \\ Shao, Ran, 15 \\ Shen, Chuan, $1 \mathrm{~F}$ \\ Shi, Aiye, 09 \\ Shi, Daming, 05 \\ Shi, Zhifeng, OF
}


Son, Dong Oh, 13

Spence, Ivor, 15

Su, Fong-Chin, OJ, OL

Sun, Qiaoyu, 05

Sun, Shiyan, OX

Sun, Yung-Nien, OJ, OL

Talebi, Ardeshir, OK

Tian, Feng, 05

Tian, Jiangxue, 04

Tian, Xiaofei, 12

Traumann, Andres, 02

Tsai, Chung-Hsien, OR

Vadiveloo, Mogana, 0 I

Vozenilek, Vit, OV

Walia, Ekta, OT

Wang, Bin, OU

Wang, Chunyue, 17

Wang, Jun, 05

Wang, Lixue, 17

Wang, Qian, OP

Wang, Qingwen, OB

Wang, Shuenn-Jyi, OR

Wang, Suyu, OE

Wang, Ting, OD

Wang, Yang, 18

Wang, Yaxing, 04

Wang, Yi, 00

Wang, Yuanyuan, OF, 19

Wei, Sui, IF

Wu, Po-Ting, OJ, OL

WU, Ying, OE

Xie, Zhifeng, OP

$\mathrm{XU}$, Guangluan, 18

$X U$, Wenhai, OU

Yan, Yaping, 07

Yang, Hua, OS, 11, 14, 1B

Yang, Shuo, 14

Yu, Jinhua, OF, 19

Yu, Xiaohao, OW

Yuan, Ding, OX, 0Z, 10

Yun, Woo-han, ON

Zhang, Bing, 1B

Zhang, Bo, OZ

Zhang, Buyang, OS, 14

Zhang, Cheng, IF

Zhang, Dinghua, 12

Zhang, Fen, $1 \mathrm{~F}$

Zhang, Hong, OX, 0Z, 10

Zhang, Hongjuan, 07

Zhang, Hua, 12

Zhang, Junping, 16

Zhang, Junru, 17

Zhang, Li, OP

Zhang, Zongxiang, OE

Zhao, Ming, OU

Zheng, Ning, 15

Zhou, Xingshe, OW

Zhou, Yuan, IE

Zhu, Jiang, OP 


\section{Conference Committee}

Advisory Committee

Godfried T. Toussaint, New York University Abu Dhabi

(United Arab Emirates)

Shaikh A. Fattah, Bangladesh University of Engineering and

Technology (Bangladesh)

Conference Chairs

Yulin Wang, Wuhan University (China)

Xudong Jiang, Nanyang Technological University (Singapore)

David Zhang, Hong Kong Polytechnic University (Hong Kong, China)

Program Committee

Vit Vozenilek, Palacky University (Czech Republic)

Hui Yu, University of Portsmouth (United Kingdom)

Tuan D. Pham, Linkoping University (Sweden)

Session Chairs

1 Image Enhancement and Pattern Recognition

Yulin Wang, Wuhan University (China)

$2 \quad$ Image Processing

Shaikh A. Fattah, Bangladesh University of Engineering and Technology (Bangladesh)

3 Image Segmentation

Hui Yu, University of Portsmouth (United Kingdom)

4 Feature Extraction and Target Detection

Kar-Ann Toh, Yonsei University (Korea)

5 Machine Vision and Visualization

Vit Vozenilek, Palacky University (Czech Republic)

6 Algorithms and Applications

Robert Kooij, TNO / Technische Universiteit Delft (Netherlands) 
Proc. of SPIE Vol. $9817981701-10$

Downloaded From: https://www.spiedigitallibrary.org/conference-proceedings-of-spie on 25 Apr 2023 Terms of Use: https://www.spiedigitallibrary.org/terms-of-use 


\title{
Introduction
}

\author{
Dear Distinguished Delegates and Guests,
}

It was a great pleasure for us to organize the Seventh International Conference on Graphic and Image Processing (ICGIP 2015) from 23-25 October 2015 in Singapore.

This conference was hosted at the Quality Hotel, Singapore, and was co-sponsored by Wuhan University and The International Association of Computer Science and Information Technology. The ICGIP conference has become a popular annual event, attracting young researchers in particular. It has established itself as a world-class conference in which participants from academia and industry interact through formal presentations and informal discussions, accompanied by keynote speakers.

The purpose of ICGIP 2015 is to share an opportunity for researchers to introduce recent issues related to Graphic and Image Processing. It covers a broad range of topics in the field, such as image acquisition, pattern recognition and analysis, signal noise control, watermarking, and nonlinear signals and systems. We thank the members of the ICGIP 2015 committee for managing the review of submissions. We believe these are the main driving factors attributing to our success.

This year 126 papers were submitted, and all the papers included in this proceedings have been peer reviewed by reviewers from the scientific committee, external reviewers, and editorial board depending on the subject matter of the paper. Reviews and initial selection were undertaken electronically. After the rigorous peer-review process, the submitted papers were selected on the basis of originality, significance, and clarity for the purpose of the conference. We want to thank all of the authors for submitting their innovative and sound work to ICGIP 2015.

Apart from technical paper presentations, there were also keynote lectures. We are grateful that the keynote speakers accepted our invitation. They were: Professor Tuan D. Pham from Linkoping University, Sweden, Professor Godfried T. Toussaint from New York University, United Arab Emirates, Professor Wang Yulin from Wuhan University, China, Professor Xudong Jiang from Nanyang Technological University, Singapore, Professor Shaikh A. Fattah from Bangladesh University of Engineering and Technology, Bangladesh, and Doctor Hui Yu from the University of Portsmouth, United Kingdom. 
We extend our gratitude to the partners and sponsors who made the events feasible. We truly believe the participants found the discussions fruitful, and that they enjoyed the opportunity to set up future collaborations. It is our sincere hope that ICGIP will one day become the leading conference in this specific academic area.

Yulin Wang Xudong Jiang 